\title{
Co-morbidade e mortalidade de pacientes em início de diálise
}

\author{
Co-morbity and mortality of patients in dialysis treatment
}

Co-morbilidad y mortalidad de pacientes al inicio de la diálisis

\section{Dulce Aparecida Barbosa ${ }^{1}$, Clara Kimiyo Gunji² ${ }^{2}$ Ana Rita de Cássia Bittencourt', Angélica Gonçalves Silva Belasco', Solange Diccini', Fátima Vattimo ${ }^{3}$, Lucila Amaral carneiro Vianna ${ }^{4}$}

\section{RESUMO}

Objetivos: identificar a co-morbidade e causas de mortalidade dos pacientes em início de diálise e analisar se as variáveis pessoais, a comorbidade, os resultados laboratoriais, o número de dias de internação e número de sessões de diálise são fatores de risco para mortalidade. Métodos: foram incluídos no estudo 102 pacientes que iniciaram tratamento dialítico em um hospital universitário. Foi realizado um registro prospectivo de dados pessoais, laboratoriais, de morbidade e mortalidade. Resultados: a hipertensão e as infecções foram as principais causas de co-morbidade (58,8\%). O sítio mais freqüente de infecção foi a corrente sangüínea em 30 (50\%). Conclusão: a maioria dos pacientes que chegaram ao Serviço estava em urgência dialítica, o sítio de infecção mais freqüente foi a corrente sangüínea relacionado ao uso do cateter venoso central, o sexo masculino, a raça branca, a uremia e as morbidades hipertensão arterial e infecção, foram as que mais contribuíram para o aumento do risco de mortalidade.

Descritores: Insuficiência renal crônica/mortalidade; Diálise renal; Mortalidade

\begin{abstract}
Objectives: to identify to the co-morbity and causes of mortality of the patients in beginning of dialysis and to analyze if the personal variables, the co-morbity, the laboratory results, the number of days of internment and number of dialysis sessions are factors of risk for mortality. Methods: was included in this study 102 patients who had initiated dialysis treatment in a university hospital. The prospective record was realized to collect personals data, laboratory data, morbity and mortality. Results: the arterial hypertension and the infection was more frequent causes of co-morbity (58,8\%). The infection locality more frequent was the blood circulation in $30(50 \%)$. Conclusion: the majority of the patients who had arrived at the service was in dialysis urgency; the morbity most prevalent was the arterial hypertension and the infection; the infection locality more frequent was the blood circulation related to the use of the central venous catheter; and that the male sex, the white race, the uremia, and the morbity arterial hypertension and infection, had been the ones that more contributed for the increase of the mortality risk.
\end{abstract}

Descriptors: Renal insufficiency, chronic/mortality ; Renal dialysis; Morbidity

\section{RESUMEN}

Objetivos: identificar el co-morbidad y las causas de la mortalidad de los pacientes en el principio de la diálisis y analizar si resultan las variables personales, el co-morbidad, los dados laboratoriais, del número de días de la internación y del número de las sesiones de la diálisis son factores del riesgo para la mortalidad. Métodos: fueron incluido 102 pacientes en el estudio que habían iniciado el tratamiento de la diálisis en un hospital de la universidad. Fue realizado uno registro de los dados personales, laboratoriais, de la morbidad y de la mortalidad. Resultados: la hipertensión arterial y las infecciónes eram las más frecuente causas de la co-morbidad (58,8\%). El local de la infección más frecuente era la circulación del sangre en 30 (50\%).Conclusão: la mayoría de los pacientes que habían llegado el servicio estaban en urgencia de la diálisis; el morbidad más frecuente era el hipertensión arterial y la infección; el local de la infección más frecuente era la circulación del sangre relacionada con el uso del catéter venoso central; y que el sexo masculino, la raza blanca, la uremia y las morbidad la hipertensión arteriale y la infección, habían sido los ese contribuido más para el aumento del riesgo de la mortalidad.

Descriptores: Insuficiência renal crônica/ mortalidad ; Diálisis renal; Morbilidad

\footnotetext{
1 Professor Adjunto da Disciplina de Fundamentos de Enfermagem e Enfermagem Médico-Cirúrgica do Departamento de Enfermagem da Universidade Federal de São Paulo - UNIFESP - São Paulo (SP), Brasil.

${ }^{2}$ Mestre em Enfermagem pelo Departamento de Enfermagem da Universidade Federal de São Paulo - UNIFESP - São Paulo (SP), Brasil..

${ }^{3}$ Doutora em Ciências da Escola de Enfermagem da Universidade de São Paulo - USP - São Paulo (SP), Brasil.

${ }^{4}$ Professor Titular do Departamento de de Enfermagem da Universidade Federal de São Paulo - UNIFESP - São Paulo (SP), Brasil.
} 


\section{INTRODUÇÃO}

Nas últimas décadas, medidas sanitárias específicas como o controle e erradicação de grandes epidemias, saneamento básico, avanço da antibioticoterapia e da quimioterapia entre outras, resultaram em acentuada redução da mortalidade por causas infecciosas e parasitárias, contribuindo para o aumento da esperança de vida e envelhecimento da população. Simultaneamente, as mudanças nos hábitos de vida e, em particular, o progressivo aumento da industrialização e urbanização têm feito com que as doenças crônico-degenerativas assumam um papel de destaque na saúde da população ${ }^{(1)}$.

As doenças crônicas são representadas por um grupo que, de modo geral, apresenta uma história natural prolongada. Caracterizam-se por período longo de latência e curso assintomático prolongado, envolvimento de múltiplos fatores de risco com participação importante do ambiente.

Além disso, são doenças que acarretam alterações patológicas irreversíveis, com conseqüente incapacidade residual. Entre estas, encontra-se a Insuficiência Renal Crônica (IRC) que pode ser referida como um diagnóstico sindrômico de perda progressiva e irreversível da função renal de depuração ${ }^{(2)}$. Em sua fase mais avançada (IRC Terminal), os rins não conseguem mais manter a normalidade do meio interno do indivíduo.

Pacientes portadores de IRC em fase terminal apresentam uma série de sinais e sintomas que configuram o quadro clínico de uremia. A fisiopatologia desse processo mórbido envolve a perda da capacidade de excreção de solutos tóxicos pelo rim, incapacidade de manter o equilíbrio hidroeletrolítico e ácido-básico do organismo, também efetuado pelo rim e alterações hormonais sistêmicas.

Quando diagnosticada a IRC Terminal, deve ser instituído um tratamento conservador ou dialítico o mais precocemente possível, caso contrário, a ocorrência de complicações pode levar à morte. A diálise é um processo de terapia renal substitutiva utilizada para remover líquidos e produtos do metabolismo corporal final ${ }^{(3)}$.

Segundo censo realizado pela Sociedade Brasileira de Nefrologia em janeiro de 2005, com 498 centros de diálise, estima-se que haja 54.311 pacientes em diálise, sendo $89 \%$ em hemodiálise, $6,7 \%$ em diálise peritoneal ambulatorial contínua, 3,8\% em diálise peritoneal automatizada e 0,4\% em diálise peritoneal intermitente ${ }^{(4)}$.

Durante o tratamento dialítico, a co-morbidade dos pacientes é elevada e está relacionada a doenças cardiovasculares, hipertensão arterial sistêmica, anemia, suscetibilidade à infecção, elevada prevalência de infecção pelo vírus da hepatite tipo B e C, doenças ósseas, desnutrição e outras causas menos definidas.

A mortalidade por IRC é 10 a 20 vezes maior que a da população geral, mesmo quando ajustada para idade, sexo, raça e presença de diabetes, sendo que a doença cardiovascular é a causa mais comum de óbito ${ }^{(5)}$. Estudo realizado em Sorocaba, São Paulo, mostrou que as causas de óbito de pacientes com IRC estão relacionadas principalmente às infecções (48\%) e doenças cardiovasculares $(40 \%)^{(\sigma)}$.

\section{RELEVÂNCIA DO ESTUDO}

O Hospital São Paulo é um hospital universitário de grande porte (690 leitos) que presta assistência terciária, com alguns pólos de assistência em nível quaternário. Considerando que se trata de uma instituição de ensino e de referência, que atende a uma demanda espontânea da população, recebendo pacientes de diversas regiões de São Paulo e muitas vezes até de outras regiões do Brasil, cada vez mais, grande número destes pacientes que chegam ao pronto socorro estão em urgência dialítica e só então são diagnosticados renais crônicos, sem nunca terem sido anteriormente tratados.

Estes pacientes apresentam níveis elevados de uréia, creatinina, potássio, hipervolemia associada, na maioria das vezes sem acesso dialítico, sendo assim, pacientes propensos a elevada morbidade e maior risco de mortalidade.

Como as características sócio-demográficas, as causas de morbidade e mortalidade nesta população de pacientes em início de diálise são desconhecidas, acreditamos que os resultados desta investigação poderão trazer contribuição clinica importante para o tratamento e prevenção das complicações que acometem estes pacientes e por isso ficamos motivadas para a realização deste estudo.

\section{OBJETIVOS}

- Identificar as causas de co-morbidade e mortalidade dos pacientes em início de diálise.

- Analisar se as variáveis pessoais; as referentes à comorbidade; os resultados laboratoriais; os dias de internação e o número de sessões de diálise dos pacientes que sobreviveram e dos que evoluíram para óbito são fatores de risco para mortalidade.

\section{MÉTODOS}

O estudo foi realizado na unidade de diálise do Hospital São Paulo, Disciplina de Nefrologia, Universidade Federal de São Paulo, no período de julho de 1999 a janeiro de 2000.

Trata-se de um estudo epidemiológico descritivo, com delineamento de Coorte controlado.

Foram incluídos no estudo todos os pacientes $(n=102)$ 
com diagnóstico de IRCT que neste período iniciaram tratamento dialítico no Hospital São Paulo.

Foi realizado um registro prospectivo de dados pessoais: nome, sexo, idade, cor, procedência, renda, hábitos (tabagismo e uso de bebida alcoólica), nível de instrução e tratamento anterior fornecidos pelos pacientes. O tipo de diálise, de acesso dialítico, tempo de diálise e número de sessões de diálise realizadas, co-morbidade associada ao tratamento e o destino dos pacientes (transferência, óbito) e nos casos de óbito, a causa do mesmo, foram registrados pelos pesquisadores. Foram coletados dos prontuários os resultados dos exames laboratoriais: creatinina, uréia, potássio, albumina, hematócrito e hemoglobina, sorologia para hepatite $\mathrm{B}$ e C e HIV no início do programa.

A realização deste estudo foi precedida da aprovação pela Comissão de Ética em Pesquisa da Universidade Federal de São Paulo (UNIFESP). Foi obtido o consentimento livre e esclarecido por escrito de todos os pacientes que participaram do estudo. Análise estatística: Para a análise estatística dos dados obtidos foram utilizados os seguintes testes: Para comparação das variáveis contínuas foram utilizados os testes " $\mathrm{t}$ " de Student ou Mann-Whitney; para comparar proporções foram usados o teste do quiquadrado ou o teste exato de Fisher. Em todos os testes fixamos em 0,05 ou $5 \%(p<0,05)$ o nível para indicar as significâncias estatísticas, assinaladas com asterisco os valores significantes. Para análise de risco de mortalidade foram calculadas as razões de risco (Risco Relativo, RR) e os respectivos intervalos de confiança em nível de $95,0 \%$ entre os pacientes que evoluíram para óbito e os que sobreviveram.

\section{RESULTADOS}

As características sócio-demográficas, as comorbidades associadas, os sítios de infecção, o destino e as causas de óbitos dos 102 pacientes com IRC, em início de diálise estão apresentados na Tabela 1.

Conforme os dados apresentados na Tabela 1, a mediana da idade dos pacientes que iniciaram diálise no período do estudo foi de 45 anos com variação de 18 a 80 anos.

Quanto ao nível de instrução e a renda familiar mensal, dos 102 pacientes, $86(84,3 \%)$ tinham apenas o ensino fundamental incompleto e a mediana da renda mensal foi de $\mathrm{R} \$ 120,00$ mensais.

A maioria dos pacientes $(80,4 \%)$ referiu não ingerir nenhum tipo de bebida alcoólica e 36,3\% eram tabagistas.

Com relação às co-morbidades, podemos observar que a hipertensão e as infecções constituem as causas de co-morbidade que prevalecem $(58,8 \%)$, seguido por diabetes mellitus em $20(19,6 \%)$ dos pacientes em início de diálise que participaram deste estudo.
Tabela 1 - Características sócio-demográficas, co-morbidades associadas, sítios de infecção, destino e causas de óbitos dos pacientes com diagnóstico de IRC em início de diálise.

\begin{tabular}{|c|c|c|}
\hline Variáveis & $\begin{array}{c}\mathrm{N} \\
\text { (mediana/ } \\
\text { variação) }\end{array}$ & $\%$ \\
\hline Idade & $\begin{array}{l}45 \text { anos } \\
(18-80)\end{array}$ & \\
\hline \multicolumn{3}{|l|}{ Sexo } \\
\hline Feminino & 53 & 51,9 \\
\hline Masculino & 49 & 48,1 \\
\hline \multicolumn{3}{|l|}{ Cor } \\
\hline Branca & 50 & 49,1 \\
\hline Não Branca & 52 & 50,9 \\
\hline \multicolumn{3}{|l|}{ Co-Morbidade* } \\
\hline Hipertensão arterial sistêmica & 60 & 58,8 \\
\hline Infecções & 60 & 58,8 \\
\hline Diabetes mellitus & 20 & 19,6 \\
\hline Cardiovasculares & 16 & 15,6 \\
\hline Autoimune & 10 & 9,8 \\
\hline Trombose venosa & 5 & 4,9 \\
\hline Doença pulmonar obstrutiva crônica & 2 & 1,9 \\
\hline Neoplasias & 2 & 1,9 \\
\hline HIV & 1 & 0,95 \\
\hline \multicolumn{3}{|l|}{ Sítio de Infecção } \\
\hline Corrente sangüínea & 30 & 50,0 \\
\hline Pulmão & 14 & 23,4 \\
\hline Peritôneo & 8 & 13,3 \\
\hline Trato urinário & 8 & 13,3 \\
\hline \multicolumn{3}{|l|}{ Destino } \\
\hline Transferidos & 70 & 68,7 \\
\hline Óbitos & 22 & 21,5 \\
\hline Continuaram no programa & 10 & 9,8 \\
\hline \multicolumn{3}{|l|}{ Causas do óbito } \\
\hline Infecciosas & 14 & 63,6 \\
\hline Metabólicas & 4 & 18,2 \\
\hline Cardiovascular & 4 & 18,2 \\
\hline
\end{tabular}

* Alguns apresentaram mais de uma morbidade.

Os resultados foram expressos em $\mathrm{n}^{\circ}=$ número de pacientes/ eventos e \% ou mediana e variação.

Dos 60 pacientes que desenvolveram infecção $(58,5 \%)$, o sítio mais freqüente foi a corrente sangüínea em $30(50 \%)$, geralmente relacionada ao cateter venoso central para hemodiálise; a infecção pulmonar esteve presente em 14 $(23,4 \%)$; a do trato urinário e a peritonite bacteriana ocorram em $8(13,3 \%)$ destes pacientes, respectivamente.

Quanto ao tipo de tratamento dialítico realizado e via de acesso utilizada nestes pacientes em início de diálise, observamos que para a grande maioria dos pacientes (75-73,5\%) o tratamento dialítico foi a hemodiálise, em $16(15,7 \%)$ a diálise peritoneal intermitente (DPI) e em $11(10,8 \%)$ a diálise peritoneal ambulatorial contínua (CAPD). Assim, a via de acesso utilizada foi, principalmente, o cateter venoso central para hemodiálise em 75 pacientes $(73,5 \%)$, enquanto nos demais, 27 (26,5\%) foi o cateter de Tenckhoff para DPI e CAPD.

Nos primeiros seis meses de tratamento dialítico, 22 $(21,5 \%)$ dos pacientes estudados evoluíram para óbito. A principal causa foi a infecção generalizada, que ocorreu 
em 14 pacientes (63,6\%). As causas metabólicas, assim como as cardiovasculares, ocorreram em apenas quatro $(18,2 \%)$ dos pacientes. Os eventos cardiovasculares foram o Acidente Vascular Cerebral e Infarto Agudo do Miocárdio com igual freqüência $(9,1 \%)$.

A análise comparativa dos dados relativos aos pacientes que sobreviveram e que evoluíram para óbito, em relação à idade, sexo, raça, exames laboratoriais, comorbidade, dias de internação e número de sessões de diálise encontram-se na Tabela 2.

Tabela 2 - Comparação da mediana de idade, sexo, raça, exames laboratoriais, co-morbidade, dias de internação e número de sessões de diálise de pacientes com diagnóstico de IRC em início de diálise, que evoluíram para óbito e sobreviveram.

\begin{tabular}{|c|c|c|c|}
\hline Variáveis & Evolução & & \\
\hline & Óbito & Sobrevida & $\begin{array}{l}\text { RR } \\
\text { (IC }\end{array}$ \\
\hline Idade & $\mathrm{n}=22$ & $\mathrm{n}=80$ & $95 \%)$ \\
\hline $\begin{array}{l}\text { mediana (variação) } \\
\text { Sexo }\end{array}$ & $39(24$ a 65$)$ & $42(18$ a 80$)$ & \\
\hline Masculino & $14 *(63,6)$ & $35(43,7)$ & \\
\hline $\begin{array}{l}\text { Feminino } \\
\text { Cor }\end{array}$ & $8(36,3)$ & $45(56,2)$ & \\
\hline Branca & $15^{*}(68,1)$ & $35(43,7)$ & \\
\hline $\begin{array}{l}\text { Não Branca } \\
\text { Exames - média } \pm \text { DP }\end{array}$ & $7(31,8)$ & $45(56,2)$ & \\
\hline Uréia (mg/dl) & $\begin{array}{c}182,59 \pm \\
80,6\end{array}$ & $\begin{array}{c}168,66 \pm \\
56,28\end{array}$ & \\
\hline Creatinina $(\mathrm{mg} / \mathrm{dl})$ & $9,42 \pm 6,38$ & $9,76 \pm 6,56$ & \\
\hline Hematócrito (\%) & $24,6 \pm 4,6^{*}$ & $28,2 \pm 7,8$ & \\
\hline Hemoglobina $(\mathrm{g} / \mathrm{dl})$ & $7,67 \pm 1,48^{*}$ & $8,42 \pm 2,45$ & \\
\hline Potássio (mEq/dl) & $5,1 \pm 1,25$ & $5,1 \pm 1,16$ & \\
\hline Albumina (g/dl) & $2,9 \pm 0,2^{*}$ & $3,9 \pm 0,5$ & \\
\hline Co-morbidade & & & \\
\hline $\begin{array}{l}\text { Hipertensão Arterial } \\
\text { Sistêmica }\end{array}$ & $20^{* *}(19,6)$ & $40(39,2)$ & $\begin{array}{l}7(1,73 \\
-28,4)\end{array}$ \\
\hline Infecção & $14(13,7)$ & $46(45,0)$ & $\begin{array}{c}1,2 \\
(0,56- \\
2,66)\end{array}$ \\
\hline Diabetes Melitus (DM) & $4(3,9)$ & $10(9,9)$ & $\begin{array}{c}1,4 \\
(0,55- \\
3,52)\end{array}$ \\
\hline Arteriopatia & $2 * *(2,0)$ & & $\begin{array}{c}7,3 \\
(0,69- \\
7,75)\end{array}$ \\
\hline $\begin{array}{l}\text { Sorologia (HbSAg } \\
\text { e/ou HCV e/ou HIV) }\end{array}$ & $6(5,9)$ & $19(18,6)$ & $\begin{array}{c}1,15 \\
(0,52- \\
2,52)\end{array}$ \\
\hline $\begin{array}{l}\text { Dias de Internação - } \\
\text { mediana (Variação) }\end{array}$ & $15(1$ a 90$)$ & $18(1$ a 120$)$ & \\
\hline $\begin{array}{l}\text { Sessões de diálise - } \\
\text { mediana (Variação) }\end{array}$ & $4(1$ a 24$)$ & $6(2$ a 48$)$ & \\
\hline
\end{tabular}

$*_{\mathrm{p}}<0,05{ }^{*} \mathrm{p}<0,001$

Não foi encontrada diferença estatisticamente significante na mediana de idade entre os pacientes que evoluíram para óbito e os que sobreviveram. Houve associação entre o sexo e a evolução para óbito, sendo que o risco relativo de mortalidade nos pacientes do sexo masculino se mostrou maior em relação ao sexo feminino $(R R=1,45$; IC 95\%, 0,97 - 2,17).

O número de óbitos ocorridos nos pacientes de cor branca foi significantemente maior do que o ocorrido entre os não brancos $(p<0,05)$. Assim como o risco relativo de mortalidade nos pacientes da raça branca foi 1,5 vez maior em relação aos da raça não branca $(R R=$ 1,56; IC 95\%, 1,06 - 2,27).

A média e o desvio padrão das dosagens de albumina, hematócrito e hemoglobina foram significantemente menores no grupo de pacientes que evoluíram para óbito em relação aos que sobreviveram $(\mathrm{p}<0,05)$.

Os testes estatísticos mostraram que o número de pacientes com hipertensão arterial que evoluíram para óbito foi significantemente maior $(\mathrm{p}<0,001)$ do que o observado no grupo sem hipertensão. $\mathrm{O}$ risco relativo de mortalidade nos pacientes com hipertensão foi sete vezes maior em relação ao grupo sem hipertensão (RR $=7)$.

Embora não tenha sido encontrada significância estatística entre o número de pacientes que evoluíram para óbito por infecção em relação aqueles que não apresentaram infecção, observou-se um risco relativo de mortalidade nos infectados de 1,2 vez maior do que em outros pacientes que foram a óbito por outras causas. Este dado sugere que a infecção foi um fator de risco importante nesta população de pacientes. Da mesma forma, não houve significância estatística em relação à mortalidade dos pacientes com e sem diabetes embora o risco de morte tenha sido 1,4 vez maior no grupo com diabetes.

A mortalidade nos pacientes com arteriopatia foi significantemente maior $(\mathrm{p}=0,04)$ sendo o risco relativo de morte sete vezes maior $(\mathrm{RR}=7,3)$. Também foi observado, que os pacientes com anemia que não receberam transfusão sangüínea apresentaram um risco relativo de mortalidade de 1,7 em relação aos que sobreviveram e foram transfundidos.

\section{DISCUSSÃO}

Quanto aos dados demográficos dos pacientes que iniciaram diálise no período do estudo, vale comentar que a mediana da idade foi de 45 anos com variação de 18 a 80 anos. Este resultado representa um dado social significativo, pois a doença crônica atinge geralmente, uma população em plena idade economicamente ativa, gerando gastos nas áreas sociais devido às aposentadorias precoces, gastos ambulatoriais e medicamentosos ${ }^{(1)}$.

O nível de instrução e a renda familiar mensal mostraram-se baixos na maioria da população deste estudo, visto que dos 102 pacientes, $86(84,3 \%)$ tinham apenas o ensino fundamental incompleto e a mediana da renda mensal foi de $\mathrm{R} \$ 120,00$ mensais, menor do que um salário mínimo, o que de certa forma poderá 
repercutir na aquisição de material e medicamentos para o controle da doença.

Neste estudo, 80,4\% dos pacientes referiram não ingerir nenhum tipo de bebida alcoólica e 36,3\% referiram o hábito de fumar. Estudo realizado sugere que o tabagismo piora a sobrevida de pacientes em hemodiálise, que diminui $72 \%$ em dois anos e $40 \%$ em seis anos nos fumantes; quando acompanhado de hipertensão arterial, estes dados são ainda mais alarmantes ${ }^{(7)}$.

A hipertensão arterial representou a principal causa associada à insuficiência renal crônica, estando presente em mais da metade $60(58,8 \%)$ dos pacientes estudados, enquanto que as infecções, têm sido apontadas na literatura como a segunda causa de co-morbidade e mortalidade dos pacientes com IRC precedida apenas dos eventos cardiovasculares. Fatores predisponentes como estresse, alimentação inadequada, fumo, uso de hormônios estrogênicos, obesidade, sedentarismo e falta de controle de saúde fazem com que o aumento da pressão arterial se torne inevitável ${ }^{(8)}$.

Em relação ao diabetes, de acordo com os resultados do presente estudo, $20(19,6 \%)$ dos pacientes em início de diálise apresentavam diabetes mellitus. Estes resultados estão de acordo com um estudo realizado nos Estados Unidos $^{(9)}$, onde $20 \%$ de todos os pacientes iniciando diálise são portadores desta doença. $\mathrm{Na}$ Grande São Paulo, em 1991, o diabetes foi a terceira causa mais freqüente de doença de base em pacientes de diálise ( $8 \%$ ), precedida por glomerulonefrite e nefroesclerose hipertensiva: $25,7 \%$ e $16,8 \%$, respectivamente ${ }^{(10)}$. A mortalidade e co-morbidade são substancialmente altas nos diabéticos mantidos em diálise, quando comparados aos não diabéticos com doença cardiovascular ${ }^{(11)}$.

Diversos fatores têm sido responsáveis pela elevada incidência de infecção em pacientes em diálise. Estes fatores estão associados ao processo dialítico, principalmente à obtenção de acesso vascular e peritoneal, às múltiplas transfusões e as más condições de higiene dos pacientes, além da imunossupressão associada à uremia ${ }^{(12)}$.

Estes dados são alarmantes e indicam a necessidade de educação continuada para os profissionais da área de saúde quanto à prevenção de infecções, tendo em vista a resposta imunológica deprimida pela própria doença. Se esta prática for adotada, deverá ocorrer a redução da morbimortalidade que acomete esta população de pacientes.

Embora a instalação do cateter venoso central seja uma técnica bastante utilizada para o estabelecimento rápido e temporário de via de acesso, é um procedimento invasivo, conhecido como importante fator de risco para desenvolvimento de infecção ${ }^{(12)}$. A condição da doença renal crônica indica a eleição de um acesso permanente que otimize a hemodiálise, oferecendo um fluxo sanguíneo adequado e que resista ao uso repetitivo por muitos anos. O ideal é que o paciente, ao entrar no programa de diálise tenha um acesso definitivo, que pode ser fístula artério venosa, porém quando ocorre dificuldade para a confecção desta, pode ser utilizada a prótese artério venosa ou o cateter venoso central permanente (permcath).

Nos Estados Unidos da América, entre 1993 e 1995, ao final do primeiro ano de tratamento dialítico, a taxa de mortalidade global em pacientes em diálise foi de 235/ 1000 pacientes ano, e destes óbitos 33\% ocorrerem por infecção ${ }^{(13)}$. Desta forma, a infecção é a maior causa de co-morbidade e a segunda causa mais freqüente de mortalidade renal crônica terminal.

Já na Europa, ao contrário do observado neste estudo, a percentagem de óbitos por causa infecciosa é menor enquanto que por eventos cardiovasculares é maior ${ }^{(14)}$.

Segundo dados do inquérito epidemiológico em unidades de diálise no Brasil, realizado em janeiro de 2000, o número de pacientes em programas de diálise no Brasil era de 47.063, sendo que $90 \%$ em hemodiálise e $10 \%$ em diálise peritoneal. A taxa de mortalidade bruta em 1999 foi de 19,7\% ${ }^{(15)}$. Estes dados mostram que a taxa de mortalidade global entre os pacientes do presente estudo, $21,5 \%$, são muito próximas as do registro brasileiro acima citado.

Os avanços tecnológicos são extremamente importantes, mas não são suficientes para a melhoria da sobrevida dos pacientes submetidos ao tratamento dialítico ${ }^{(16)}$.

Estudo realizado nos Estados Unidos da América verificou que a mortalidade nos pacientes no sexo masculino foi significantemente maior em relação a pacientes do sexo feminino, e da mesma forma significantemente maior nos pacientes brancos em relação aos não brancos. Estes autores atribuem este fato a maior freqüência dos eventos cardiovasculares fatais nos pacientes do sexo masculino da cor branca ${ }^{(17)}$. Em nosso estudo, o número de óbitos ocorridos nos pacientes da raça branca foi significantemente maior do que o ocorrido entre os não brancos $(p<0,05)$. Assim como o risco relativo de mortalidade nos pacientes da raça branca foi 1,5 vez maior em relação aos da raça não branca $(R R=$ 1,56; IC $95 \%, 1,06$ - 2,27).

A albumina é muito importante na manutenção do volume do líquido no sistema vascular, já que as paredes dos capilares são impermeáveis à albumina, sua presença no plasma determina elevação da pressão oncótica que favorece a manutenção do fluido no espaço intravascular, contribuindo para elevação do fluido circulante efetivo e conseqüentemente, em condições cardíacas adequadas, à estabilidade hemodinâmica. A albumina, que é produzida no fígado, tem a capacidade de ligar-se a inúmeras substâncias presentes no plasma, funcionando como proteína de transporte para metais, ácidos graxos, bilirrubinas, drogas, entre outras substâncias ${ }^{(18)}$.

Outro estudo evidenciou que a albumina sérica foi significantemente menor em pacientes que evoluíram para óbito em até 90 dias do início do tratamento dialítico quando comparados àqueles que morreram mais 
tardiamente. Este dado sugere que a mortalidade precoce nos pacientes em início de tratamento pode estar relacionada ao déficit nutricional ${ }^{(18)}$.

A anemia, principal conseqüência hematológica da insuficiência renal, constitui o fator responsável pela limitação da capacidade física dos pacientes renais crônicos, prejudicando sua reabilitação social e profissional e acarretando uma qualidade de vida insatisfatória ${ }^{(19)}$.

A origem da anemia renal é a deficiência de eritropoetina, devido à redução da massa renal, e comprometimento na síntese renal deste hormônio. A função dos glóbulos vermelhos é, principalmente, a de transporte de oxigênio dos pulmões para os tecidos e um dos principais constituintes da hemoglobina é o ferro e a eritropoietina contribui para a maturação da hemácia tornando-a funcionalmente capacitada para esta atividade.

\section{CONCLUSÕES}

- A hipertensão e a infecção constituíram as causas mais prevalentes de co-morbidade em pacientes em início de diálise.

- A mortalidade de $21,5 \%$ entre os pacientes estudados foi considerada elevada, sendo a infecção a causa mais freqüente de mortalidade $(63,6 \%)$.

- A dosagem de albumina, hematócrito e hemoglobina nos pacientes que evoluíram para óbito foi significantemente menor $(\mathrm{p}<0,05)$ em relação à dos que sobreviveram.

- O risco relativo de morte por infecção foi $20 \%$ maior no grupo que teve infecção.

- Os pacientes com anemia que não receberam transfusão sangüínea apresentaram um risco relativo de morte de 1,7 vezes maior em relação aos que sobreviveram e receberam transfusão.

- O sítio de infecção mais freqüente foi a corrente sangüínea, relacionado ao uso do cateter venoso central, via de acesso mais utilizada nestes pacientes.

- O risco relativo de morte nos pacientes do sexo masculino foi duas vezes maior em relação ao sexo feminino. Nos pacientes da cor branca em relação aos não brancos foi 1,5 vez maior. $\mathrm{O}$ risco de mortalidade por hipertensão foi sete vezes maior em relação ao grupo dos não hipertensos.

- A educação continuada dos profissionais de saúde visando à prevenção de infecção deve ser adotada para redução da morbi-mortalidade.

\section{REFERÊNCIAS}

1. Lessa I. Doenças crônicas degenerativas. In: Rouquayrol MZ. Epidemiologia \& saúde. 5a ed. Rio de Janeiro: Medsi; 1999. p. 411-5.

2. Romão Júnior JE. Doença renal crônica: definição, epidemiologia e classificação. J Bras Nefrol. 2004; 26 (3 Supl 1):1-3.

3. Draibe SA. Diálise crônica. In: Prado FC, Ramos JA, Valle
JR. Atualização terapêutica: manual prático de diagnóstico e tratamento. 22a ed. São Paulo: Artes Médicas; 2005. p.929-32.

4. Sociedade Brasileira de Nefrologia. Censo 2004/2005 [texto na Internet]. São Paulo:SBN; c2005. [citado 2005 Out 10]. Disponível em: URL: $<$ http://www.sbn.org.br.

5. Teixeira VPC, Abreu PF, Deus RB, Kirsztajn GM. Progressão de doença renal e sua prevenção. In: Prado FC, Ramos JA, Valle JR. Atualização terapêutica: manual prático de diagnóstico e tratamento. 22a ed. São Paulo: Artes Médicas; 2005. p.904-05.

6. D'Avila R, Guerra EMM, Rodrigues CIS, Fernandes FA, Cadaval RAM, Almeida FA. Sobrevida de pacientes renais crônicos em diálise peritoneal e hemodiálise. J Bras Nefrol. 1999; 21(1): 13-21.

7. Biernat JC. Sobrevida em hemodiálise. In: Cruz J, Barros RT, Sesso RCC, David Neto E, Suassuna JHR, Heilberg IP, Gouvêa Filho WL, coordenadores. Atualidades em nefrologia 3. São Paulo: Savier; 1994. p. 155-9.

8. Pinheiro ME, Alves CMP. Hipertensão arterial na diálise e no transplante renal. J Bras Nefrol. 2003; 25(3):142-8.

9. Faria JBL. Tatamento conservador de nefropatia diabética. In: Cruz J, Barros RT, Sesso RCC, David Neto E, Suassuna JHR, Heilberg IP, Gouvêa Filho WL, coordenadores. Atualidades em nefrologia 3. São Paulo: Savier; 1994. p.202-10.

10. Sesso R, Anção MS, Madeira SA. Aspectos epidemiológicos do tratamento dialítico na Grande São Paulo. Rev Assoc Med Bras (1992). 1994; 40(1):10-4.

11. Beltrame RE, Guilhen JC, Araújo IM, Zanolli MB. Nefropatia em pacientes diabéticos $\mathrm{X}$ nefropatia em pacientes não diabéticos. Análise de 221 pacientes tratados no Hospital das Clinicas da Faculdade de Medicina de Marilia. RBM Rev Bras Med. 2004; 61(5): 298:302-300-304.

12. Cendoroglo M Neto, Draibe SA. Intercorrências infecciosas no paciente urêmico. In: Prado FC, Ramos JA, Valle JR. Atualização terapêutica: manual prático de diagnóstico e tratamento. 22a ed. São Paulo: Artes Médicas; 2005. p. 935-8.

13. Vanholder R, Massy Z, Argiles A, Spasovski G, Verbeke F, Lameire N; European Uremic Toxin Work Group. Chronic kidney disease as cause of cardiovascular morbidity and mortality. Nephrol Dial Transplant. 2005; 20(6):1048-56. Epub 2005 Apr 6.

14. Us Renal Data System. Annual Data Report. Bethhesda (MD.): National Institutes of Health, National Institute of Diabetes and Kidney Diseases; 1997.

15. Sesso R. Inquérito epidemiológico em unidade de diálise do Brasil. J Bras Nefrol. 2000, 22 (3 Supl 2):23-6.

16. Andrade LGM, Gabriel DP, Martin LC, Cruz AP, Balbi AL, Caramori JT, Barretti P. Sobrevida em hemodiálise no hospital das clinicas da faculdade de medicina de Botucatu Unesp: comparação entre a primeira e a segunda metades da década de 90. J Bras Nefrol. 2005; 27(1):1-7.

17. Bloembergen WE, Port FK, Mauger EA, Wolfe RA. Causes of death in dialysis patients: racial and gender differences. J Am Soc Nephrol. 1994; 5(5):1231-42.

18. Santos NSJ, Draibe SA, Kamimura MA, Cuppari L. Albumina sérica como marcador nutricional de pacientes em hemodiálise. Rev Nutr. 2004; 17(3):339-49.

19. Stevens P. Optimizing renal anaemia management-benefits of early referral and treatment. Nephrol Dial Transplant. 2005; 20 Suppl 8: viii22-26. 